\title{
An overdetermined system with two super-singular lines containing second order hyperbolic equations
}

\author{
N. Rajabov, A. N. Adib, and Mohamed Elsayed
}




\title{
AN OVERDETERMINED SYSTEM WITH TWO SUPER-SINGULAR LINES CONTAINING SECOND ORDER HYPERBOLIC EQUATIONS
}

\author{
N. RAJABOV, A. N. ADIB, AND MOHAMED ELSAYED
}

Received January 1, 2010

\begin{abstract}
The paper treats a linear overdetermined system which contains second order hyperbolic equations with two linear super singularities. The authors obtain some new integral representations of the solutions.
\end{abstract}

2000 Mathematics Subject Classification: 35-XX; 35. H05; 35125

Keywords: hyperbolic equations, boundary value problems, system of linear equations, integral representations, supper singularities

\section{INTRODUCTION}

We derive the general solutions of the system (2.1) that contains arbitrary constants subject to the conditions $\alpha<1, \beta<1, \gamma<1$ or $\alpha>1, \beta>1, \gamma>1$.

Hyperbolic differential equations with singular coefficients or singular surfaces possess importance in diverse areas of mathematical physics and mathematical engineering, including elasticity, hydrodynamics, thermodynamics and other problems. Furthermore, it is also well known that hyperbolic differential equations with one or more singular lines occur in engineering and physical processes. For example, the non-standard hyperbolic equation of second order with two singular lines is employed to describe the transformation spectrum of electric signals on long lines with variable parameters in the theory of the electric flail [3,4,9].

The problem of obtaining the solutions of equations of hyperbolic type with singular coefficients is described in a number of works [1,3-5,9]. In [9], for the equation

$$
\frac{\partial^{2} U}{\partial t^{2}}=\sum_{j=1}^{n}\left[a_{j} \frac{\partial^{2} U}{\partial x_{j}^{2}}+\frac{\eta_{j}}{X_{j}} \frac{\partial U}{\partial x_{j}}\right]+g(x, t)
$$

(here $a_{j}=$ const. $>o, \eta_{j}=$ const. $>o$ ) a representation of the solutions was obtained by using the solutions of the corresponding regularity equation. On this basis a solution of the Cauchy problem was received, when the boundary conditions are 
given on the $t=o$ surface. In [3], the equation

$$
\frac{\partial^{2} U}{\partial t^{2}}+\frac{\eta}{t} \frac{\partial U}{\partial t}-\left(\frac{\partial^{2} U}{\partial x^{2}}+\frac{\partial^{2} U}{\partial y^{2}}\right)=f(x, y, t) .
$$

(in the case when $\eta>0$ in region $x>0, t>0,-\infty<y<\infty$ ) was solved

$$
U(x, 0)=0,\left.\frac{\partial U(x, y, t)}{\partial t}\right|_{t=0}=0, U(0, y, t)=g(y, t) .
$$

Investigations of first and second order overdetermined systems with singular and super-singular coefficients are described in $[6,10]$. A study of a nonlinear system of ordinary differential equations with singular and super-singular coefficient (in a case which is connected to the theory of P-Laplacians and psedo-Laplacians) is described in [8].

\section{MAin Results}

Let $D$ be the following region: $D=\left\{0<x<\lambda_{1}, 0<y<\lambda_{2}\right\}$, which is bounded by

$$
\Gamma_{1}=\left\{0<x<\lambda_{1}, y=0\right\}, \quad \Gamma_{2}=\left\{x=0,0<y<\lambda_{2}\right\} .
$$

In the region $D$ we consider the following system:

$$
\left\{\begin{array}{l}
\frac{\partial u(x, y)}{\partial x \partial y}+\frac{a_{1}(x, y)}{y^{\beta}} \frac{\partial u(x, y)}{\partial x}+\frac{b_{1}(x, y)}{x^{\alpha}} \frac{\partial u(x, y)}{\partial y}+\frac{c_{1}(x, y)}{x^{\alpha} y^{\beta}} u(x, y)=\frac{f_{1}(x, y)}{x^{\alpha} y^{\beta}} \\
\frac{\partial u(x, y)}{\partial y}+\frac{b_{2}(x, y)}{y^{\gamma}} u(x, y)=\frac{f_{2}(x, y)}{y^{\gamma}}
\end{array}\right.
$$

where the coefficients $a_{1}(x, y), b_{j}(x, y), c_{1}(x, y), f_{j}(x, y), j=1,2$, are given functions in the domain $\bar{D}$. Moreover $\alpha=$ const. $>0, \beta=$ const. $>0, \gamma=$ const. $>0$.

2.1. The case of $\alpha<1, \beta<1, \gamma<1$.

We first determine an integral representation of the solution of the given system in the case when the coefficients of the first equation are related together in a certain way. Then we investigate the case when the coefficients of the first equation of system (2.1) are not related to each other.

Let $C_{y}^{1}(\bar{D})$ denote the set functions which are continuous on $\bar{D}$ together with their derivatives with respect to the variable $y$.

We always assume that $b_{1}(x, y) \in C_{y}^{1}(\bar{D})$ and that the coefficients $a_{1}(x, y)$, $b_{1}(x, y), b_{2}(x, y), c_{1}(x, y)$ are continuous functions in the domain $D$.

According to [9], the first equation of system (2.1) can be written in the following form

$$
\left(\frac{\partial}{\partial y}+\frac{a_{1}(x, y)}{y^{\beta}}\right)\left(\frac{\partial}{\partial x}+\frac{b_{1}(x, y)}{x^{\alpha}}\right) u(x, y)=\frac{f_{1}(x, y)+c_{2}(x, y) u(x, y)}{x^{\alpha} y^{\beta}},
$$

where

$$
c_{2}(x, y)=y^{\beta} \frac{\partial b_{1}(x, y)}{\partial y}+a_{1}(x, y) \cdot b_{1}(x, y)-c_{1}(x, y)
$$




\subsubsection{The case of $c_{2}(x, y)=0$}

Suppose that the functions $a_{1}(x, y), b_{1}(x, y)$ and $c_{1}(x, y)$ of the system (2.1) satisfy the following relation

$$
c_{1}(x, y)=y^{\beta} \frac{\partial b_{1}(x, y)}{\partial y}+a_{1}(x, y) \cdot b_{1}(x, y)
$$

for all $(x, y) \in D$.

Under this condition, the first equation of (2.1) can be written in the following form:

$$
\left(\frac{\partial}{\partial y}+\frac{a_{1}(x, y)}{y^{\beta}}\right)\left(\frac{\partial}{\partial x}+\frac{b_{1}(x, y)}{x^{\alpha}}\right) u(x, y)=\frac{f_{1}(x, y)}{x^{\alpha} y^{\beta}} .
$$

The solution of this equation is

$$
\begin{aligned}
u(x, y) & =\exp \left[-\Omega_{b_{1}}^{\alpha}(x, y)\right]\left[\psi_{1}(y)+\int_{0}^{x} \exp \left[\Omega_{b_{1}}^{\alpha}(t, y)-\Omega_{a_{1}}^{\beta}(t, y)\right] \cdot\left(\varphi_{1}(t)+\right.\right. \\
& \left.\left.+\int_{0}^{y} \exp \left[\Omega_{a_{1}}^{\beta}(t, s)\right] \cdot t^{-\alpha} s^{-\beta} \cdot f_{1}(t, s) d s\right) d t\right] \equiv K_{1}\left[\varphi_{1}(x), \psi_{1}(y), f_{1}(x, y)\right],
\end{aligned}
$$

where $\varphi_{1}(x)$ and $\psi_{1}(y)$ are arbitrary continuous functions belonging to the class $C^{1}\left(\Gamma_{1}\right)$ and $C^{1}\left(\Gamma_{2}\right)$, respectively. In fact, these functions will be determined in the sequel,

$$
\Omega_{b_{1}}^{\alpha}(x, y)=\int_{0}^{x} \frac{b_{1}(t, y)}{t^{\alpha}} d t, \quad \Omega_{a_{1}}^{\beta}(x, y)=\int_{0}^{y} \frac{a_{1}(x, s)}{s^{\beta}} d s .
$$

Now, we write the second equation of (2.1) in the following form:

$$
\frac{\partial}{\partial y}\left[\exp \left[\Omega_{b_{2}}^{\gamma}(x, y)\right] \cdot u(x, y)\right]=\exp \left[\Omega_{b_{2}}^{\gamma}(x, y)\right] y^{-\gamma} f_{2}(x, y),
$$

where

$$
\Omega_{b_{2}}^{\gamma}(x, y)=\int_{0}^{y} \frac{b_{2}(x, s)}{s^{\gamma}} d s .
$$

By plugging $u(x, y)$ from (2.3) into (2.4) we obtain

$$
\begin{aligned}
& \frac{\partial}{\partial y}\left\{\operatorname { e x p } [ \Omega _ { b _ { 2 } } ^ { \gamma } ( x , y ) - \Omega _ { b _ { 1 } } ^ { \alpha } ( x , y ) ] \left[\psi_{1}(y)+\int_{0}^{x} \exp \left[\Omega_{b_{1}}^{\alpha}(t, y)-\Omega_{a_{1}}^{\beta}(t, y)\right] \cdot\left(\varphi_{1}(t)+\right.\right.\right. \\
& \left.\left.\left.+\int_{0}^{y} \exp \left[\Omega_{a_{1}}^{\beta}(t, s)\right] t^{-\alpha} s^{-\beta} f_{1}(t, s) d s\right) d t\right]\right\}=f_{2}(x, y) y^{-\gamma} \exp \left[\Omega_{b_{2}}^{\gamma}(x, y)\right] .
\end{aligned}
$$

Suppose that the functions $b_{1}(x, y)$ and $b_{2}(x, y)$ satisfy the following relation

$$
y^{\gamma} \frac{\partial b_{1}(x, y)}{\partial y}=x^{\alpha} \frac{\partial b_{2}(x, y)}{\partial x} .
$$


By differentiating and using the relation (2.6), the equality (2.5) can be expressed in the form

$$
\begin{aligned}
& \psi^{\prime}{ }_{1}(y)+\frac{b_{2}(0, y)}{y^{\gamma}} \psi_{1}(y)=f_{2}(x, y) y^{-\gamma} \cdot \exp \left[\Omega_{b_{1}}^{\alpha}(x, y)\right]-\frac{b_{2}(0, y)}{y^{\gamma}} \\
& \cdot \int_{0}^{x} \exp \left[\Omega_{b_{1}}^{\alpha}(t, y)-\Omega_{a_{1}}^{\beta}(t, y)\right] \cdot\left(\varphi_{1}(t)+\int_{0}^{y} \exp \left[\Omega_{a_{1}}^{\beta}(t, s)\right] t^{-\alpha} s^{-\beta} f_{1}(t, s) d s\right) d t- \\
& -\int_{0}^{x} \frac{\partial}{\partial y}\left\{\exp \left[\Omega_{b_{1}}^{\alpha}(t, y)-\Omega_{a_{1}}^{\beta}(t, y)\right] \cdot\left(\varphi_{1}(t)+\int_{0}^{y} \exp \left[\Omega_{a_{1}}^{\beta}(t, s)\right] t^{-\alpha} s^{-\beta} f_{1}(t, s) d s\right)\right\} d t
\end{aligned}
$$

Since the left hand side does not depend on $\mathrm{x}$, we have

$$
\begin{aligned}
& \frac{\partial}{\partial x}\left[f_{2}(x, y) y^{-\gamma} \exp \left[\Omega_{b_{1}}^{\alpha}(x, y)\right]\right]-\frac{\partial}{\partial y}\left\{\operatorname { e x p } [ \Omega _ { b _ { 1 } } ^ { \alpha } ( x , y ) - \Omega _ { a _ { 1 } } ^ { \beta } ( x , y ) ] \cdot \left(\varphi_{1}(x)+\right.\right. \\
& \left.\left.+\int_{0}^{y} \exp \left[\Omega_{a_{1}}^{\beta}(x, s)\right] x^{-\alpha} s^{-\beta} f_{1}(x, s) d s\right)\right\}=b_{2}(0, y) y^{-\gamma} \exp \left[\Omega_{b_{1}}^{\alpha}(x, y)-\Omega_{a_{1}}^{\beta}(x, y)\right] \\
& \cdot\left(\varphi_{1}(x)+\int_{0}^{y} \exp \left[\Omega_{a_{1}}^{\beta}(x, s)\right] x^{-\alpha} s^{-\beta} f_{1}(x, s) d s\right),
\end{aligned}
$$

or in a simpler form

$$
\begin{aligned}
& x^{\alpha} \frac{\partial f_{2}(x, y)}{\partial x}+b_{1}(x, y) f_{2}(x, y)=\left(b_{2}(x, y)-a_{1}(x, y) y^{\gamma-\beta}\right) \exp \left[-\Omega_{a_{1}}^{\beta}(x, y)\right] . \\
& \cdot\left[x^{\alpha} \varphi_{1}(x)+\int_{0}^{y} \exp \left[\Omega_{a_{1}}^{\beta}(x, s)\right] s^{-\beta} f_{1}(x, s) d s\right]+f_{1}(x, y) y^{\gamma-\beta},(x, y) \in D,
\end{aligned}
$$

Making use of (2.7), we can define $\psi_{1}(y)$ by the following ordinary differential equation

$$
\psi^{\prime}{ }_{1}(y)+\frac{b_{2}(0, y)}{y^{\gamma}} \psi_{1}(y)=\frac{f_{2}(0, y)}{y^{\gamma}} .
$$

According to [6,9], the solution of this equation is represented by the following form

$$
\psi_{1}(y)=\exp \left[-\Omega_{b_{2}}^{\gamma}(0, y)\right]\left[C+\int_{0}^{y} \exp \left[\Omega_{b_{2}}^{\gamma}(0, s)\right] s^{-\gamma} f_{2}(0, s) d s\right],
$$

where $C$ is an arbitrary constant.

From expression (2.8), the function $x^{\alpha} \varphi_{1}(x)$ can be expressed in the form

$$
\begin{aligned}
& x^{\alpha} \varphi_{1}(x)=\frac{x^{\alpha} \frac{\partial f_{2}(x, y)}{\partial x}+b_{1}(x, y) f_{2}(x, y)-f_{1}(x, y) y^{\gamma-\beta}}{b_{2}(x, y)-a_{1}(x, y) y^{\gamma-\beta}} \cdot \exp \left[\Omega_{a_{1}}^{\beta}(x, y)\right]- \\
& -\int_{0}^{y} \exp \left[\Omega_{a_{1}}^{\beta}(x, s)\right] s^{-\beta} f_{1}(x, s) d s,\left(b_{2}(x, y) \neq a_{1}(x, y) y^{\gamma-\beta}\right) .
\end{aligned}
$$


We note that the left part of this expression is independent of the variable y, so we have

$$
\begin{aligned}
& \frac{\partial}{\partial y}\left\{\frac{x^{\alpha} \frac{\partial f_{2}(x, y)}{\partial x}+b_{1}(x, y) f_{2}(x, y)-f_{1}(x, y) y^{\gamma-\beta}}{b_{2}(x, y)-a_{1}(x, y) y^{\gamma-\beta}} \cdot \exp \left[\Omega_{a_{1}}^{\beta}(x, y)\right]\right\}= \\
& =\exp \left[\Omega_{a_{1}}^{\beta}(x, y)\right] y^{-\beta} f_{1}(x, y) .
\end{aligned}
$$

By using (2.11) and assuming that the following limit exists,

$$
\lim _{y \rightarrow 0}\left\{\frac{x^{\alpha} \frac{\partial f_{2}(x, y)}{\partial x}+b_{1}(x, y) f_{2}(x, y)-f_{1}(x, y) y^{\gamma-\beta}}{b_{2}(x, y)-a_{1}(x, y) y^{\gamma-\beta}} \cdot \exp \left[\Omega_{a_{1}}^{\beta}(x, y)\right]\right\}=F_{1}(x),
$$

the function $\varphi_{1}(x)$ can be determined by the following formula:

$$
\varphi_{1}(x)=F_{1}(x) x^{-\alpha}, \quad \alpha<1, \quad F_{1}(x) \in C\left(\overline{\Gamma_{1}}\right) .
$$

In the particular case of $\gamma=\beta$, if $y \rightarrow 0$, then

$$
\varphi_{1}(x)=x^{-\alpha}\left[\frac{x^{\alpha} \frac{\partial f_{2}(x, 0)}{\partial x}+b_{1}(x, 0) f_{2}(x, 0)-f_{1}(x, 0)}{b_{2}(x, 0)-a_{1}(x, 0)}\right]
$$

from equality (2.10).

After simplification, condition (2.11) can be written in the following form:

$$
\begin{gathered}
x^{\alpha}\left[b_{2}(x, y)-a_{1}(x, y) y^{\gamma-\beta}\right] \frac{\partial^{2} f_{2}(x, y)}{\partial x \partial y} \\
+\left\{\frac{a_{1}(x, y)}{y^{\beta}}\left[b_{2}(x, y)-a_{1}(x, y) y^{\gamma-\beta}\right] x^{\alpha}-\right. \\
\left.x^{\alpha} \frac{\partial}{\partial y}\left[b_{2}(x, y)-a_{1}(x, y) y^{\gamma-\beta}\right]\right\} \frac{\partial f_{2}(x, y)}{\partial x}+ \\
b_{1}(x, y)\left[b_{2}(x, y)-a_{1}(x, y) y^{\gamma-\beta}\right] \frac{\partial f_{2}(x, y)}{\partial y}+ \\
+\left\{\frac{a_{1}(x, y)}{y^{\beta}} b_{1}(x, y) \cdot\left[b_{2}(x, y)-a_{1}(x, y) y^{\gamma-\beta}\right]-\right. \\
b_{1}(x, y) \frac{\partial}{\partial y}\left[b_{2}(x, y)-a_{1}(x, y) y^{\gamma-\beta}\right]+ \\
\left.+\left[b_{2}(x, y)-a_{1}(x, y) y^{\gamma-\beta}\right] \cdot \frac{\partial b_{1}(x, y)}{\partial y}\right\} f_{2}(x, y) \\
=y^{\gamma-\beta}\left[b_{2}(x, y)-a_{1}(x, y) y^{\gamma-\beta}\right] \frac{\partial f_{1}(x, y)}{\partial y}+ \\
+\left\{\left[b_{2}(x, y)-a_{1}(x, y) y^{\gamma-\beta}\right]^{2} y^{-\beta}\right.
\end{gathered}
$$




$$
\begin{array}{r}
+\frac{a_{1}(x, y)}{y^{\beta}}\left[b_{2}(x, y)-a_{1}(x, y) y^{\gamma-\beta}\right] y^{\gamma-\beta}-y^{\gamma-\beta} \frac{\partial}{\partial y}\left[b_{2}(x, y)-\right. \\
\left.\left.-a_{1}(x, y) y^{\gamma-\beta}\right]+(\gamma-\beta) y^{\gamma-\beta-1} \cdot\left[b_{2}(x, y)-a_{1}(x, y) y^{\gamma-\beta}\right]\right\} f_{1}(x, y),
\end{array}
$$

where $(x, y) \in D$.

In what the follows, $C_{x y}^{2}(D)$ will denote the set of all functions $u(x, y)$ that are continuous on $D$ together with their first derivatives, and $u_{x y} \in C(D)$.

Henceforth, we have the following conclusion:

Theorem 1. Let $\alpha<1, \beta<1, \gamma<1$ in system (2.1), and assume that the coefficients and right hand sides of this system satisfy the following conditions:

1) $a_{1}(x, y), b_{2}(x, y), f_{2}(x, y) \in C_{x}^{1}(\bar{D}), b_{1}(x, y), f_{1}(x, y) \in C_{y}^{1}(\bar{D}), f_{2}(x, y) \in$ $C_{x y}^{\prime}(D), \frac{\partial}{\partial y}\left[b_{2}(x, y)-a_{1}(x, y) y^{\gamma-\beta}\right] \in C(D)$,

2) $c_{1}(x, y)=y^{\beta} \frac{\partial b_{1}(x, y)}{\partial y}+a_{1}(x, y) \cdot b_{1}(x, y)$,

3) $y^{\gamma} \frac{\partial b_{1}(x, y)}{\partial y}=x^{\alpha} \frac{\partial b_{2}(x, y)}{\partial x}$,

4) The functions $f_{1}(x, y)$ and $f_{2}(x, y)$ satisfy conditions (2.8), (2.1.1),

5) The limit (2.12) exists.

Then the desired solution of system (2.1) of class $C_{x y}^{2}(D)$ is determined by (2.3), in which $\varphi_{1}(x)$ which is determined by formula (2.13), where the value of $\psi_{1}(y)$ is defined by formula (2.9), provided that $C$ is an arbitrary constant.

Example 1. Assume that in (2.1) $\alpha<1, \beta<1, \gamma<1, \beta=\gamma$ and the coefficients are constants, moreover, suppose that the right parts are defined by the following functions:

$$
\begin{gathered}
f_{1}(x, y)=x^{\alpha} \exp \left[b_{1} \omega_{\alpha}(x)+a_{1} \omega_{\beta}(y)\right], \\
f_{2}(x, y)=x \exp \left[b_{1} \omega_{\alpha}(x)+a_{1} \omega_{\beta}(y)\right] E(y) .
\end{gathered}
$$

where

$$
E(y)=1-\left(b_{2}-a_{1}\right) \omega_{\beta}(y), \quad \omega_{\beta}(y)=\frac{1}{(\beta-1) y^{\beta-1}}, \quad \omega_{\alpha}(x)=\frac{1}{(\alpha-1) x^{\alpha-1}} .
$$

Then the function of the form

$$
u(x, y)=c \exp \left[b_{2} \omega_{\gamma}(y)+b_{1} \omega_{\alpha}(x)\right]-x \omega_{\beta}(y) \exp \left[a_{1} \omega_{\beta}(y)+b_{1} \omega_{\alpha}(x)\right]
$$

is the solution of system (2.1), where $c$ is an arbitrary constant .

$$
\text { 2.1.2. } c_{2}(x, y) \neq 0
$$

Suppose that the functions $a_{1}(x, y), b_{1}(x, y), c_{1}(x, y)$ satisfy the following relation

$$
c_{1}(x, y) \neq y^{\beta} \frac{\partial b_{1}(x, y)}{\partial y}+a_{1}(x, y) \cdot b_{1}(x, y)
$$


for all $(x, y) \in D$ in the system (2.1).

In this case, the first equation of system (2.1), can be represented in the form (2.2). We denote $f_{3}(x, y)=f_{1}(x, y)+c_{2}(x, y) u(x, y)$ where $f_{3}(x, y)$ is a given function. Then the solution of equation (2.2) can be written as

$$
u(x, y)=K_{1}\left[\varphi_{1}(x), \psi_{1}(y), f_{3}(x, y)\right] .
$$

Using these items that are dependent on $u(x, y)$, we obtain the following integral equation:

$$
\begin{aligned}
u(x, y)- & \int_{0}^{x} d t \int_{0}^{y}\left[\exp \left[\Omega_{b_{1}}^{\alpha}(t, y)-\Omega_{b_{1}}^{\alpha}(x, y)+\Omega_{a_{1}}^{\beta}(t, s)-\Omega_{a_{1}}^{\beta}(t, y)\right] .\right. \\
& \cdot \frac{c_{2}(t, s)}{t^{\alpha} s^{\beta}} u(t, s) d s=K_{1}\left[\varphi_{1}(x), \psi_{1}(y), f_{1}(x, y)\right] .
\end{aligned}
$$

In fact, the problem of finding the solution of the overdetermined system (2.1), in the case of $\alpha<1, \beta<1, \gamma<1$, is reduced to the solution of a two dimensional Volterra integral equation with two weak singular lines in the kernel (2.17).

According to $[7,11]$, the solution of the integral equation (2.17) can be represented in the following form

$$
\begin{gathered}
u(x, y)=K_{1}\left[\varphi_{1}(x), \psi_{1}(y), f_{1}(x, y)\right]+ \\
\int_{0}^{x} d t \int_{0}^{y} \Gamma(x, y ; t, s) K_{1}\left[\varphi_{1}(t), \psi_{1}(s), f_{1}(t, s)\right] d s \\
\equiv T_{1,1}\left[\varphi_{1}(x), \psi_{1}(y), f_{1}(x, y)\right],
\end{gathered}
$$

where $\Gamma(x, y ; t, s)$ is the resolvent of the integral equation (2.17).

We now return to the problem of finding the arbitrary functions $\varphi_{1}(x), \psi_{1}(y)$. We repeat the scheme of 2.1.1 in the case when $\gamma=\beta$, and $y \rightarrow 0$. We obtain

$$
\begin{gathered}
\varphi_{1}(x)=x^{-\alpha}\left[\frac{x^{\alpha} \frac{\partial f_{2}(x, 0)}{\partial x}+b_{1}(x, 0) f_{2}(x, 0)-f_{1}(x, 0)-c_{2}(x, 0) u(x, 0)}{b_{2}(x, 0)-a_{1}(x, 0)}\right], \\
\left(b_{2}(x, 0) \neq a_{1}(x, 0)\right), \\
\psi_{1}(y)=\exp \left[-\Omega_{b_{2}}^{\gamma}(0, y)\right]\left[C+\int_{0}^{y} \exp \left[\Omega_{b_{2}}^{\gamma}(0, s)\right] s^{-\gamma} f_{2}(0, s) d s\right], \\
\psi_{1}(0)=C, \\
u(x, 0)=\exp \left[-\Omega_{b_{1}}^{\alpha}(x, 0)\right]\left[\psi_{1}(0)+\int_{0}^{x} \exp \left[\Omega_{b_{1}}^{\alpha}(t, 0)\right] \varphi_{1}(t) d t\right] .
\end{gathered}
$$

By substituting the value of $\varphi_{1}(x)$ from (2.19) into (2.21), we obtain the following one dimensional Volterra type integral equation: 


$$
\begin{aligned}
& u(x, 0)+\int_{0}^{x} \exp \left[\Omega_{b_{1}}^{\alpha}(t, 0)-\Omega_{b_{1}}^{\alpha}(x, 0)\right] \cdot \frac{c_{2}(t, 0) u(t, 0)}{b_{2}(t, 0)-a_{1}(t, 0)} \frac{d t}{t^{\alpha}}=\exp \left[-\Omega_{b_{1}}^{\alpha}(x, 0)\right] \\
& \cdot\left\{\psi_{1}(0)+\int_{0}^{x} \exp \left[\Omega_{b_{1}}^{\alpha}(t, 0)\right]\left[\frac{t^{\alpha} \frac{\partial f_{2}(t, 0)}{\partial t}+b_{1}(t, 0) f_{2}(t, 0)-f_{1}(t, 0)}{b_{2}(t, 0)-a_{1}(t, 0)}\right] \frac{d t}{t^{\alpha}}\right\}, \quad(2.22)
\end{aligned}
$$

Take a new unknown function

$$
W(x)=u(x, 0) \exp \left[\Omega_{b_{1}}^{\alpha}(x, 0)\right] .
$$

Then it follows that the integral equation (2.22) can be represented in the following form

$$
W(x)+\int_{0}^{x} \frac{c_{2}(t, 0) W(t)}{b_{2}(t, 0)-a_{1}(t, 0)} \frac{d t}{t^{\alpha}} d t=E(x), \alpha<1,
$$

where

$$
E(x)=\left\{\psi_{1}(0)+\int_{0}^{x} \exp \left[\Omega_{b_{1}}^{\alpha}(t, 0)\right]\left[\frac{t^{\alpha} \frac{\partial f_{2}(t, 0)}{\partial t}+b_{1}(t, 0) f_{2}(t, 0)-f_{1}(t, 0)}{b_{2}(t, 0)-a_{1}(t, 0)}\right] \frac{d t}{t^{\alpha}}\right\},
$$

and $\alpha<1$. According to [2], the solution of equation (2.24) is given by the following formula

$$
W(x)=E(x)-\int_{0}^{x} \exp \left[\Omega_{c_{3}}^{\alpha}(t, 0)-W_{c_{3}}^{\alpha}(x, 0)\right] \frac{c_{3}(t)}{t^{\alpha}} E(t) d t \equiv T_{2}[E(x)],
$$

where

$$
c_{3}(x)=\frac{c_{2}(x, 0)}{b_{2}(x, 0)-a_{1}(x, 0)}, W_{c_{3}}^{\alpha}(x, 0)=\int_{0}^{x} \frac{c_{3}(t)}{t^{\alpha}} d t .
$$

Using formula (2.23), the solution of integral equation (2.22) can be written as

$$
u(x, 0)=\exp \left[-\Omega_{b_{1}}^{\alpha}(x, 0) T_{2}[E(x)] .\right.
$$

By substituting the value of $u(x, 0)$ into (2.19), we obtain the value of $\phi_{1}(x)$ :

$$
\begin{gathered}
\varphi_{1}(x)= \\
x^{-\alpha}\left[\frac{x^{\alpha} \frac{\partial f_{2}(x, 0)}{\partial x}+b_{1}(x, 0) f_{2}(x, 0)-f_{1}(x, 0)-c_{2}(x, 0) \exp \left[-\Omega_{b_{1}}^{\alpha}(x, 0)\right] T_{2}[E(x)]}{b_{2}(x, 0)-a_{1}(x, 0)}\right],
\end{gathered}
$$

Then we substitute the values of $\varphi_{1}(x), \psi_{1}(y)$ which were obtained from (2.1.2) and (2.20), respectively, in formula (2.17).

Henceforth, we deduce the following conclusion 
Theorem 2. Let $\alpha<1, \beta<1, \gamma<1, \gamma=\beta$ in system (2.1). Assume the followings: the coefficients and right hand sides of this system satisfies the conditions: $1,3,5$, from theorem 1 , and $c_{2}(x, y) \neq 0$. Moreover, suppose that the functions $f_{1}(x, y), f_{2}(x, y)$ satisfy the following conditions:

(1)

$$
\begin{gathered}
x^{\alpha} \frac{\partial f_{2}(x, y)}{\partial x}+b_{1}(x, y) f_{2}(x, y) \\
=\left(b_{2}(x, y)-a_{1}(x, y) y^{\gamma-\beta}\right) \exp \left[-W_{a_{1}}^{\beta}(x, y)\right]\left[x^{\alpha} \varphi_{1}(x)+\right. \\
+\int_{0}^{y} \exp \left[\Omega_{a_{1}}^{\beta}(x, s)\right] s^{-\beta} \\
\left.\left\{f_{1}(x, s)+c_{2}(x, s) T_{1,1}\left[\varphi_{1}(x), \psi_{1}(y), f_{1}(x, y)\right]\right\} d s\right]+ \\
+y^{\gamma-\beta}\left[f_{1}(x, y)+c_{2}(x, y) T_{1,1}\left[\varphi_{1}(x), \psi_{1}(y), f_{1}(x, y)\right],\right.
\end{gathered}
$$

(2)

$$
\begin{gathered}
x^{\alpha}\left[b_{2}(x, y)-a_{1}(x, y) y^{\gamma-\beta}\right] \frac{\partial^{2} f_{2}(x, y)}{\partial x \partial y} \\
+\left\{\frac{a_{1}(x, y)}{y^{\beta}}\left[b_{2}(x, y)-a_{1}(x, y) y^{\gamma-\beta}\right] x^{\alpha}-x^{\alpha} \frac{\partial}{\partial y}\left[b_{2}(x, y)-\right.\right. \\
\left.\left.-a_{1}(x, y) y^{\gamma-\beta}\right]\right\} \frac{\partial f_{2}(x, y)}{\partial x}+b_{1}(x, y)\left[b_{2}(x, y)-a_{1}(x, y) y^{\gamma-\beta}\right] \frac{\partial f_{2}(x, y)}{\partial y}+ \\
\left\{\frac{a_{1}(x, y)}{y^{\beta}} b_{1}(x, y)\left[b_{2}(x, y)-a_{1}(x, y) y^{\gamma-\beta}\right]-b_{1}(x, y) \frac{\partial}{\partial y}\left[b_{2}(x, y)-a_{1}(x, y) y^{\gamma-\beta}\right]\right. \\
\left.+\left[b_{2}(x, y)-a_{1}(x, y) y^{\gamma-\beta}\right] \frac{\partial b_{1}(x, y)}{\partial y}\right\} f_{2}(x, y) \\
=y^{\gamma-\beta}\left[b_{2}(x, y)-a_{1}(x, y) y^{\gamma-\beta}\right] \\
\frac{\partial}{\partial y}\left[f_{1}(x, y)+c_{2}(x, y) \cdot T_{1,1}\left[\varphi_{1}(x), \psi_{1}(y), f_{1}(x, y)\right]\right]+ \\
\left\{\left[b_{2}(x, y)-a_{1}(x, y) y^{\gamma-\beta}\right]^{2} y^{-\beta}+\frac{a_{1}(x, y)}{y^{\beta}}\right. \\
\cdot\left[b_{2}(x, y)-a_{1}(x, y) y^{\gamma-\beta}\right] y^{\gamma-\beta} \\
-y^{\gamma-\beta} \frac{\partial}{\partial y}\left[b_{2}(x, y)-a_{1}(x, y) y^{\gamma-\beta}\right]+(\gamma-\beta) y^{\gamma-\beta-1} \\
\left.\cdot\left[b_{2}(x, y)-a_{1}(x, y) y^{\gamma-\beta}\right]\right\}\left[f_{1}(x, y)+c_{2}(x, y) T_{1,1}\left[\varphi_{1}(x), \psi_{1}(y), f_{1}(x, y)\right]\right] .
\end{gathered}
$$

Then the desired solution of system (2.1) in the class $C_{x y}^{2}(D)$ is determined by (2.3), in which $\varphi_{1}(x)$ is defined by (2.1.2) and $\psi_{1}(y)$ is determined by formula (2.20) , where $C$ is an arbitrary constant. 
2.2. The case of $b_{1}(x, y) \in C_{y}^{1}(\bar{D}), \alpha>1, \beta>1, \gamma>1$.

In system (2.1) let $\alpha>1, \beta>1, \gamma>1, c_{2}(x, y)=0$. By repeating the method that was explained in section 2.1.1, we obtain the following result:

Theorem 3. Let $\alpha>1, \beta>1, \gamma>1$, in system (2.1) and assume that the coefficients and right hand sides of this system satisfy the conditions 1, 2, 3 of Theorem 1. Suppose also that $a_{1}(0,0)>0, b_{1}(0,0)>0, b_{2}(0,0)>0$. Moreover, assume the existence of the following limits:

$$
\begin{gathered}
E_{1}(x)=\lim _{y \rightarrow 0}\left\{\frac{x^{\alpha} \frac{\partial f_{2}(x, y)}{\partial x}+b_{1}(x, y) f_{2}(x, y)-f_{1}(x, y) y^{\gamma-\beta}}{b_{2}(x, y)-a_{1}(x, y) y^{\gamma-\beta}}\right. \\
\left.\exp \left[W_{a_{1}}^{\beta}(x, y)-a_{1}(0,0) \omega_{\beta}(y)\right]\right\}, \\
E_{2}(y)=\lim _{x \rightarrow 0}\left(\exp \left[-b_{1}(0,0) \omega_{\alpha}(x)\right] f_{2}(x, y)\right),
\end{gathered}
$$

where $E_{1}(x) \in C\left(\overline{\Gamma_{1}}\right), E_{2}(y) \in C\left(\overline{\Gamma_{2}}\right)$.

Besides, suppose that the functions $f_{1}(x, y), f_{2}(x, y)$ satisfy the condition (2.11) and it is also true that

$$
\begin{gathered}
x^{\alpha} \frac{\partial f_{2}(x, y)}{\partial x}+b_{1}(x, y) f_{2}(x, y) \\
=\left(b_{2}(x, y)-a_{1}(x, y) y^{\gamma-\beta}\right) \exp \left[a_{1}(0,0) \omega_{\beta}(y)-W_{a_{1}}^{\beta}(x, y)\right] \\
\left(x^{\alpha} \varphi_{2}(x)+\int_{0}^{y} \exp \left[W_{a_{1}}^{\beta}(x, s)-a_{1}(0,0) \omega_{\beta}(s)\right] s^{-\beta} f_{1}(x, s) d s\right)+f_{1}(x, y) y^{\gamma-\beta},
\end{gathered}
$$

$(x, y) \in D, \varphi_{2}(x)=E_{1}(x) x^{-\alpha}, \alpha>1, \beta>1$.

Moreover, assume that the functions, $a_{1}(x, y), b_{1}(x, y), b_{2}(x, y)$ in he neighborhoods $\Gamma_{1}$ and $\Gamma_{2}$ satisfy the following conditions:

$$
\begin{array}{lll}
\left|b_{1}(x, y)-b_{1}(0,0)\right| \leq H_{1} x^{\delta_{3}}, & \delta_{3}>\alpha-1, & \text { at } \quad x \rightarrow 0 ; \\
\left|a_{1}(x, y)-a_{1}(0,0)\right| \leq H_{2} y^{\delta_{4}}, & \delta_{4}>\beta-1, & \text { at } \quad y \rightarrow 0 ; \\
\left|b_{2}(x, y)-b_{2}(0,0)\right| \leq H_{3} x^{\delta_{5}}, & \delta_{5}>\gamma-1, & \text { at } \quad y \rightarrow 0 ;
\end{array}
$$

Then the desired solution of system (2.1) in the class $C_{x y}^{2}(D)$ is determined by:

$$
\begin{gathered}
u(x, y)=\exp \left[-W_{b_{1}}^{\alpha}(x, y)+b_{1}(0,0) \omega_{\alpha}(x)\right]\left\{\psi_{2}(y)+\right. \\
+\int_{0}^{x} \exp \left[W_{b_{1}}^{\alpha}(t, y)-b_{1}(0,0) \omega_{\alpha}(t)+a_{1}(0,0) \omega_{\beta}(y)-W_{a_{1}}^{\beta}(t, y)\right] \cdot\left(\varphi_{2}(t)+\right. \\
\left.\left.+\int_{0}^{y} \exp \left[W_{a_{1}}^{\beta}(t, s)-a_{1}(0,0) \omega_{\beta}(s)\right] \cdot t^{-\alpha} s^{-\beta} \cdot f_{1}(t, s) d s\right) d t,\right\}
\end{gathered}
$$

where

$$
\psi_{2}(y)=\exp \left[b_{2}(0,0) \omega_{\gamma}(y)-W_{b_{2}}^{\gamma}(0, y)\right][C
$$




$$
\left.+\int_{0}^{y} \exp \left[W_{b_{2}}^{\gamma}(0, s)-b_{2}(0,0) \omega_{\gamma}(s)\right] s^{-\gamma} E_{2}(s) d s\right]
$$

(here $C$ is an arbitrary constant), and

$$
\begin{gathered}
W_{b_{1}}^{\alpha}(x, y)=\int_{0}^{x} \frac{b_{1}(t, y)-b_{1}(0,0)}{t^{\alpha}} d t, W_{a_{1}}^{\beta}(x, y)=\int_{0}^{y} \frac{a_{1}(x, s)-a_{1}(0,0)}{s^{\beta}} d s, \\
W_{b_{2}}^{\gamma}(x, y)= \\
\int_{0}^{y} \frac{b_{2}(x, s)-b_{2}(0,0)}{s^{\gamma}} d s, \omega_{\gamma}(y)=\frac{1}{(\gamma-1) y^{\gamma-1}}, \omega_{\alpha}(x)=\frac{1}{(\alpha-1) x^{\alpha-1}} .
\end{gathered}
$$

Remark 2. The integral representation (3) also satisfies the system (2.1) when $b_{2}(0,0)<0, a_{1}(0,0)<0, b_{1}(0,0)<0$, and the functions $E_{2}(y), E_{1}(x)$ are such that $E_{2}(0)=0, E_{1}(0)=0$, and they have the following asymptotic behaviors:

$$
\begin{aligned}
& E_{2}(y)=0\left[\exp \left[-\left|b_{2}(0,0)\right| \omega_{\gamma}(y)\right] y^{\delta_{1}}\right], \quad \delta_{1}>\gamma-1, \quad \text { as } \quad y \rightarrow 0, \\
& E_{1}(x)=0\left[\exp \left[-\left|b_{1}(0,0)\right| \omega_{\alpha}(x)\right] \cdot x^{\delta_{2}}\right], \quad \delta_{2}<\alpha-1, \quad \text { as } \quad x \rightarrow 0 .
\end{aligned}
$$

Moreover, the function $f_{1}(x, y)$ satisfies that $f_{1}(x, 0)=0, f_{1}(0, y)=0$, and it has the following asymptotic behavior:

$$
\begin{gathered}
f_{1}(x, y)=0\left[\exp \left[-\left|a_{1}(0,0)\right| \omega_{\beta}(y)\right] \cdot y^{-\delta_{4}}\right], \quad y \rightarrow 0, \\
f_{1}(x, y)=0\left[\exp \left[-\left|b_{1}(0,0)\right| \omega_{\alpha}(x)\right] \cdot x^{-\delta_{3}}\right], \quad x \rightarrow 0 .
\end{gathered}
$$

Remark 1. If the coefficients of system (2.1) satisfy all of the conditions of Theorem 3 , then the solution of the system (2.1) is unbounded in $\Gamma_{1}, \Gamma_{2}$, and its behavior is determined by the following asymptotic conditions:

$$
u(x, y)=O\left[\exp \left(b_{1}(0,0) \omega_{\alpha}(x)\right)\right] \text { at } x \rightarrow 0,
$$

and

$$
u(x, y)=O\left[\exp \left(b_{2}(0,0) \omega_{\gamma}(y)\right)\right] \text { at } y \rightarrow 0,\left(\gamma>\beta \quad \text { or } \quad \gamma=\beta, b_{2}(0,0)>a_{1}(0,0)\right) .
$$

Remark 2. For all possible cases of the parameters $\alpha, \beta$ and $\gamma$, integral representations of the solutions of the system (2.1) are obtained not only in the case when $c_{1}(x, y)=y^{\beta} \frac{\partial b_{1}(x, y)}{\partial y}+a_{1}(x, y) \cdot b_{1}(x, y)$, but also when $c_{1}(x, y) \neq y^{\beta} \frac{\partial b_{1}(x, y)}{\partial y}+$ $a_{1}(x, y) \cdot b_{1}(x, y)$. 


\section{REFERENCES}

[1] D. Baĭnov and P. Simeonov, Integral inequalities and applications, ser. Mathematics and its Applications (East European Series). Dordrecht: Kluwer Academic Publishers Group, 1992, vol. 57.

[2] G. Bognár, M. Rontó, and N. Rajabov, "On initial value problems related to $p$-Laplacian and pseudo-Laplacian,” Acta Math. Hungar., vol. 108, no. 1-2, pp. 1-12, 2005.

[3] R. W. Carroll and R. E. Showalter, Singular and degenerate Cauchy problems, ser. Mathematics in Science and Engineering. New York-London: Academic Press [Harcourt Brace Jovanovich, Publishers], 1976, vol. 127.

[4] R. P. Gilbert, Function theoretic methods in partial differential equations, ser. Mathematics in Science and Engineering. New York-London: Academic Press, 1969, vol. 54.

[5] H. Levine, Partial differential equations, ser. AMS/IP Studies in Advanced Mathematics. Providence, RI; International Press, Cambridge, MA: American Mathematical Society, 1997, vol. 6.

[6] N. Rajabov, An introduction to the theory of partial differential equations with super-singular coefficients. Dushanbe, 1992, pp. 236 (Translated in English Tehran University, pp. 222, 1997).

[7] N. Rajabov, Volterra type integral equation with boundary and interior fixed singularity and supersingularity kernels and their application. Devashtich, Dushanbe, 2007, pp. 221.

[8] N. Rajabov, "About one class of linear first order overdetermined systems with interior singular and super-singular manifolds," in More progress in Analysis. Catantia , Italy, 25-30 July (2005), World Scientific, New Yersey-London: Proceedings of the 5th International ISAAC congress, 2009, pp. 1207-1218.

[9] N. Rajabov, Integral representations and boundary value problems for some differential equations with singular lines or singular surfaces. $\quad$ Dushanbe (Russian), Part 1, 1980-126 p., Part 2, 1981170 p., Part 3, 1982-170 p., Part 4, 1985-157 p.

[10] N. Rajabov and E. Mohamed, "About one class second order three linear overdetermined system, contain second order hyperbolic equation with two degenerating lines," Herald Tajik National University (Science series), Dushanbe, vol. 49, no. 1, pp. 37-47, 2009.

[11] N. Rajabov, M. Rontó, and L. Rajabova, "On some two dimensional Volterra type linear integral equations with super-singularity," Math. Notes (Miskolc), vol. 4, no. 1, pp. 67-76, 2003.

Authors' addresses

\section{N. Rajabov}

D.Sc. in Math., Head of the Chair of Mathematical Analysis and Function Theory, Doctor of Sciences (Mathematics), Professor, Associate member Tajik Academy of Sciences, Faculty of Mathematics, Tajik National University, Dushanbe, Tajikistan.

E-mail address: nusrat38@mail.ru

\section{A. N. Adib}

Associate Professor of Mathematics, Suez Canal University, Faculty of Petroleum and Mining Engineering, Mathematics Department, Suez, Egypt.

E-mail address: adel.nasim@yahoo.com

\section{Mohamed Elsayed}

PH.D. student, Tajik National University, Tajikistan, from Egypt, Associate Lecture, University of Banha, Faculty of Science, Department of Mathematics, Egypt

E-mail address: mohamed_el77@yahoo.com 